\title{
A stable lead halide perovskite nanocrystals protected by PMMA
}

\author{
Xiao $\mathrm{Li}^{1,2}$, Zhenjie Xue ${ }^{2}$, Dan $\mathrm{Luo}^{2}$, Chuanhui Huang ${ }^{2}$, Lizhi $\mathrm{Liu}^{2}$, Xuezhi Qiao ${ }^{2}$, Cong Liu², \\ Qian Song ${ }^{2}$, Cong $\mathrm{Yan}^{2}$, Yingchun $\mathrm{Li}^{1}$ and Tie Wang ${ }^{2^{*}}$
}

\begin{abstract}
To enhance the stability in humidity is very crucial to hybrid organic-inorganic lead halide perovskites in a broad range of applications. This report describes a coating stratergy of perovskite nanocrystals via polymethylmethacrylate-introduced ligand-assisted reprecipitation, using the interactions between the $\mathrm{Pb}$ cations on the surface of perovskite nanocrystals and the functional ester carbonyl groups in polymethylmethacrylate framework. The hydrophobic framework shields the open metal sites of hybrid organic-inorganic lead halide perovskites from being attacked by water, effectively retarding the diffusion of water into the perovskite nanocrystals. The as-prepared films demonstrate high resistance to heat and moisture. Additionally, the introduction of polymethylmethacrylate into ligand-assisted reprecipitation can effectively control the bulk precipitation and promote the stability of the perovskite solution.
\end{abstract}

Keywords: perovskite nanocrystals, polymer framework, surface coatings, interface phase

\section{INTRODUCTION}

Recently, hybrid organic-inorganic lead halide perovskites (HOIPs) have aroused extensive interest due to their potential application in a wide variety of high-performance optoelectronic devices, including solar cells [14], light-emitting diodes [5-7], optically pumped lasers [8] and photodetectors $[9,10]$. However, HOIP materials suffer from poor stability under external stresses, especially moisture, heat and light, due to their low formation energy (approximate $0.1-0.3 \mathrm{eV}$ ) [11-14]. Once water approaches the perovskite surface, it prefers to strongly bind $\mathrm{Pb}$ cations, and then the structure degrades within only 8.5 ps $[15,16]$. Enhancing the stability of the perovskite is necessary to prolong the lifetime of its devices.
The intrinsic sensitivity of perovskite to external stress can be reduced by crosslinking between the organic and inorganic moieties $[17,18]$, but hydrogen bonds between methylammounium iodide (MAI) and perovskite cannot avoid to be attacked by water. The other method to stabilize HOIPs against water is covering them with waterresistant materials including mesoporous inorganic matrices [19-22] or organic small molecules [23]. Most recently, microencapsulation of polymer was found to offer a barrier to moisture [24-27], but the methods tend to generate lead ions at the surface and the easily exfoliated coating due to the weak chemical bonds [28].

Here a coating of perovskite nanocrystals (NCs) was anchored via the ligand-assisted reprecipitation (LAR) which usually offers good surface coating on NCs $[29,30]$. To bond the $\mathrm{Pb}$ cations of perovskite NCs, polymethyl methacrylate (PMMA) was introduced on HOIP NCs by LAR method. A strong attractive interaction brings about a compact interface $[28,31,32]$, which can effectively retard the diffusion of water into NCs for the decreased intermolecular spaces. Due to the ester carbonyl groups of PMMA, the obtained composite HOIP films show good quality, pure colors and ultrahigh stability against heat and water.

\section{EXPERIMENTAL SECTION}

\section{Chemicals}

Lead(II) bromide $\left(\mathrm{PbBr}_{2}\right.$, analytical reagent, 99.0\%), oleylamine (OLAM, 70\%), oleic acid (OA, 90\%), hydrobromic acid ( $\mathrm{HBr}, 48$ wt.\% in $\left.\mathrm{H}_{2} \mathrm{O}, \geq 99.99 \%\right)$, PMMA (SU) and methylamine $\left(\mathrm{CH}_{3} \mathrm{NH}_{2}, 33\right.$ wt.\% in absolute ethanol) were purchased from Sigma-Aldrich. N,N-dimethylformamide (DMF, analytical reagent, 99.5\%), die-

\footnotetext{
${ }^{1}$ School of Materials Science and Engineering, North University of China, Taiyuan 030051, China

${ }^{2}$ Beijing National Laboratory for Molecular Sciences, Key Laboratory of Analytical Chemistry for Living Biosystems, Institute of Chemistry, Chinese Academy of Sciences, Beijing 100190, China

* Corresponding author (email: wangtie@iccas.ac.cn)
} 
thyl ether (DME, analytical reagent, 99.5\%) and toluene (Tol, analytical reagent, 99.5\%) were purchased from Beijing Chemical Works. All chemicals were used without any further purification.

Polymer introduced LAR (PLAR) synthesis of MAPbBr ${ }_{3}^{-}$ PMMA solution

$0.4 \mathrm{~mL} \mathrm{OA}$ and $4 \mathrm{~mL}$ DMF with ultrasonic agitation was heated to $40^{\circ} \mathrm{C} .0 .04 \mathrm{~mL}$ OLAM, $58.7 \mathrm{mg} \mathrm{PbBr}_{2}$ and $14.4 \mathrm{mg} \mathrm{MABr}$ were added after each step per $5 \mathrm{~min}$ to form a clear transparent solution. $0.5 \mathrm{~mL}$ precursor solution was rapidly injected into $5 \mathrm{~mL}$ toluene with $68.6 \mathrm{mg}$ PMMA preheated to $40^{\circ} \mathrm{C}$. The reaction was quenched after $2 \mathrm{~h}$ at $40^{\circ} \mathrm{C}$, and cooled to room temperature. Then a bright yellow green supernatant $\mathrm{MAPbBr}_{3}$-PMMA solution with green-emitting was gained.

\section{Preparation of PLAR composite films}

PLAR solution was processed onto the glass through cotton swab painting in air-circulating oven and dried at $40^{\circ} \mathrm{C}$ for $3 \mathrm{~h}$. A freestanding PLAR composite film was obtained by peeling off it from the glass plate.

\section{Simple nanocrystal blends with PMMA films (SNBP)}

$1 \mathrm{mg}$ LAR-synthesized $\mathrm{MAPbBr}_{3} \mathrm{NCs}$ were blended with $5 \mathrm{~mL}$ of toluene and $68.6 \mathrm{mg}$ of PMMA. The blend solution was deposited by cotton swab painting in an aircirculating oven and dried at $40^{\circ} \mathrm{C}$ for $3 \mathrm{~h}$.

\section{Sandwich structure PMMA films with an $\mathrm{MAPbBr}_{3} \mathrm{NCs}$ core (SPNC)}

$0.1 \mathrm{mg}$ LAR-synthesized $\mathrm{MAPbBr}_{3}$ NCs placed into the top surface of pure PMMA through cotton swab painting in an air-circulating oven and dried at $40^{\circ} \mathrm{C}$ for $3 \mathrm{~h}$. Afterward, the pure PMMA solution (68.6 mg PMMA dissolved in $5 \mathrm{~mL}$ toluene) was processed onto the above PMMA films through cotton swab painting and dried at $40^{\circ} \mathrm{C}$ for $3 \mathrm{~h}$.

\section{The $\mathrm{MAPbBr}_{3}$ NCs at the top surface of PMMA (NTSP) films}

LAR-synthesized $\mathrm{MAPbBr}_{3} \mathrm{NCs}$ were placed onto the top face of pure PMMA through cotton swab painting in an air-circulating oven and dried at $40^{\circ} \mathrm{C}$ for $3 \mathrm{~h}$.

\section{Characterization}

The morphology of the obtained products was determined with transmission electron microscopy (TEM) on a JEOL JEM-1011 with a thermionic gun operating at an accelerating voltage of $100 \mathrm{kV}$ and with high-resolution TEM (HRTEM) on a JEOL JEM-2100F working at an accelerating voltage of $200 \mathrm{kV}$. Powder X-ray diffraction (XRD) patterns of the obtained products were measured on a PANalytical Empyrean-2 X-ray diffractometer using a $\mathrm{Cu} \mathrm{Ka}$ source with a $1 / 2^{\circ}$ fixed diffraction slit and a $1 \mathrm{~mm}$ receiver slit. UV-vis absorbance spectra were recorded with a Shimadzu UV-1800 spectrophotometer. Photoluminescence (PL) measurements were acquired using an RF-5301PC spectrofluorophotometer. The differential scanning calorimetry (DSC) analysis was performed on a NETZSCH DSC differential scanning calorimeter under a nitrogen atmosphere. Thermogravimetric analysis (TGA) experiments were performed on a Pyris 1 TGA Perkin-Elmer Instrument (Wellesley, MA). Scanning electron microscopy (SEM) images together with energy-dispersive X-ray spectroscopy (EDS). X-ray photoelectron spectroscopy (XPS) was performed on a Thermo Scientific ESCALab 250Xi using $200 \mathrm{~W}$ monochromatic Al Ka radiation. Fourier transform infrared spectroscopy (FTIR) of the obtained products was conducted on a BRUKER TENSOR-27. Time-resolved PL (TRPL) measurements were performed on an Edinburgh Instruments FLS980 fluorescence spectrophotometer using a time-correlated single-photon counting (TCSPC) spectrometer with a pulsed laser diode (EPL360). PL quantum yields (PL-QY) were acquired using an integrating sphere incorporated into the FLS980 spectrofluorometer. The temperature-dependent PL spectra were measured on a Horiba luminescence spectrometer (HR 320) equipped with a closed-circuit liquid helium cryostat (Jannis CCS-100). Inductively coupled plasma optical emission spectroscopy (ICP-OES) was performed on a Thermo Scientific iCAP 6300.

\section{Stability tests}

For water stability test, PLAR composite films without further protection were immersed in deionized water at room temperature for three months. For nearly boiling water test at temperature of $90^{\circ} \mathrm{C}$, all films were put into nearly boiling water for 3, 5, 6, 10 and 20 min subsequently before they were taken out, and cooled down to room temperature for optical microscopy, PL, PLQY, SEM and XRD characterizations.

\section{RESULTS AND DISCUSSION}

The fabrication of $\mathrm{MAPbBr}_{3} \mathrm{NC}$ composite films is shown in a schematic illustration (Fig. 1a). Such composite films can be cut into various shapes with green luminescence (Fig. 1b). A ten-fold diluted $\mathrm{MAPbBr}_{3} \mathrm{NC}$ 
a

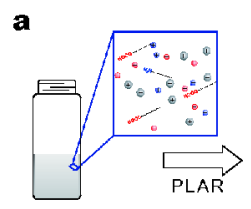

Precursor

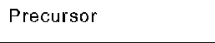

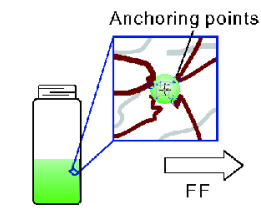

NCs in PMMA solution

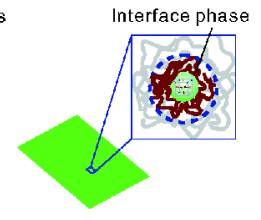

PLAR film
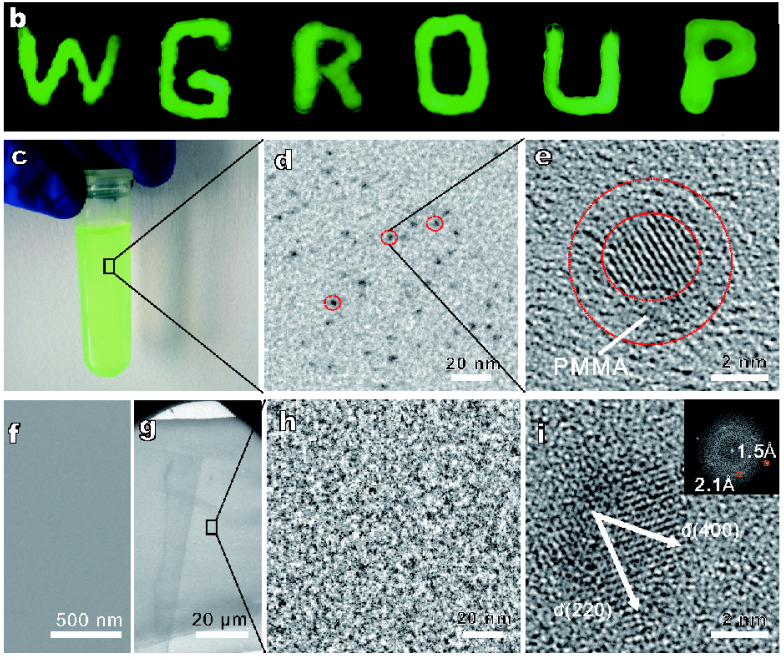

Figure 1 PLAR-synthesized MAPbBr ${ }_{3}$ NCs. (a) Schematic illustration of fabricating $\mathrm{MAPbBr}_{3}$ NC-embedded PMMA composite films; (b) luminescent photo of various shapes of cotton swab painting; (c) optical image of a ten-fold diluted $\mathrm{MAPbBr}_{3} \mathrm{NC}$-PMMA solution without any purification; (d) TEM image of $\mathrm{MAPbBr}_{3} \mathrm{NCs}$ in the supernatant of the PLAR solution after washing once; (e) HRTEM showing $\mathrm{MAPbBr}_{3} \mathrm{NCs}$ encapsulated by thin polymer shells; (f) SEM image of top surface of $\mathrm{MAPbBr}_{3}$ composite film; (g) low magnification TEM image of an ultrathin slice with a depth dimension of $50 \mathrm{~nm}$ after treatment at the low temperature of $-60^{\circ} \mathrm{C}$; (h) a typical TEM image of cross-section of PMMA films containing $\mathrm{MAPbBr}_{3}$ NCs; (i) HRTEM image of a single $\mathrm{MAPbBr}_{3} \mathrm{NC}$ in films, the inset is the corresponding FFT image.

solution shows a clear green color without any purification (Fig. 1c). The PLAR-synthesized $\mathrm{MAPbBr}_{3} \mathrm{NCs}$ with $2.3 \pm 0.5 \mathrm{~nm}$ were characterized by TEM (Fig. $1 \mathrm{~d}$, e and Fig. S1), which indicated that the PMMA uniformly coated on individual $\mathrm{MAPbBr}_{3}$ NCs. SEM represents smooth morphologies of the composite films without visible aggregations (Fig. 1f). After cutting into an ultrathin slice, no phase separation can be observed between the discrete phase $\mathrm{MAPbBr}_{3} \mathrm{NCs}$ and the continuous phase PMMA (Fig. 1g, h), which implies a good interface to stabilize $\mathrm{MAPbBr}_{3}$ NCs. As shown in the HRTEM (Fig. 1i) and fast Fourier transform (FFT) images (inset of Fig. 1i), the $\mathrm{MAPbBr}_{3} \mathrm{NCs}$ embedded in the composite films display interplanar distances of 1.5 and $2.1 \AA$, respectively, corresponding to the (400) and (220) crystal planes in the cubic phase. These NCs have a $\mathrm{Br} / \mathrm{Pb}$ molar ratio of 3:1 (Fig. S2), in accordance with the stoichiometry of

$\mathrm{MAPbBr}_{3 .}$

Without PMMA, LAR-synthesized $\mathrm{MAPbBr}_{3} \mathrm{NCs}$ have an average diameter of $3.2 \pm 0.8 \mathrm{~nm}$ (Figs S1a and S3), with a large amount of macro-size aggregations [33], accompanied by yellow-red sediments at the bottom of containers (Fig. S4). The crystalline size of the precipitates in the LAR synthesis is significantly larger than that in PLAR synthesis (Figs S5 and S6). PLAR synthesis can hence effectively control the bulk precipitation and promote the long-term stability of $\mathrm{MAPbBr}_{3} \mathrm{NCs}$. The stability plays an important role in the industrial production of the materials. To test the system stability, we left the LAR synthesis solution at room temperature under air humidity ( $90 \% \mathrm{RH})$. The color of the LAR sample disappeared entirely after 14 days (Fig. S7a), while the PLAR sample maintained bright green emission even after 90 days (Fig. S7b), which shows the best stability compared with the previous results [20].

On the anchor points, the bond between the discrete phase $\mathrm{MAPbBr}_{3}$ nanocrystal and the continuous phase PMMA in PLAR-synthesized $\mathrm{MAPbBr}_{3} \mathrm{NC}$ composite films are shown in Fig. 2a, which was characterized using FTIR. Compared with the pristine PMMA spectrum, a new absorption band of $1678 \mathrm{~cm}^{-1}$ corresponding to the asymmetric stretch of the COO group appears in PLAR films [27], indicating the bonding between the $\mathrm{C}=\mathrm{O}$ group and the surface $\mathrm{Pb}^{2+}$ (Fig. $2 \mathrm{~b}$ and Fig. S8). The average anchoring point number of polymer chain on the $\mathrm{MAPbBr}_{3} \mathrm{NCs}$ can be quantitatively determined by the FTIR spectra of pure PMMA, SNBP films and PLAR films $[26,29,30,34]$. The anchor point number of each chain in PLAR films is about twice as much as that of SNBP films (Supplementary Text S1 and Fig. 2c). Such a larger number of anchors results in a higher concentration of PMMA chains and a denser interface around the NCs, thus fewer unbonded- $\mathrm{Pb}$ ions are found on the surface. DSC of pure PMMA, SNBP films and PLAR films show the glass transition temperatures $\left(T_{\mathrm{g}}\right)$ as $T_{\mathrm{g}(\mathrm{PMMA})}>$ $T_{\mathrm{g}(\mathrm{SNBP} \text { films) }}>T_{\mathrm{g}(\mathrm{PLAR} \text { films) }}$ (Fig. S9), suggesting that the motion of molecular chain segment of PMMA was intensified by perovskite NCs because the lone pair electrons entering the empty atomic orbit of the central $\mathrm{Pb}$ ion weaken the polarity of the side chain of PMMA [35]. TGA of pure PMMA, SNBP films and PLAR films were performed from room temperature to $700^{\circ} \mathrm{C}$ under air. The PLAR films showed the highest thermal decomposition temperatures and lowest thermal decomposition rates (Fig. S10). The thermo-oxidative stability of the PLAR films is remarkably higher than that of pure PMMA, because in the PLAR films the thermal motion 
a

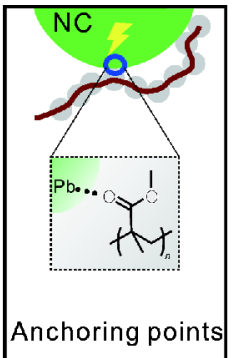

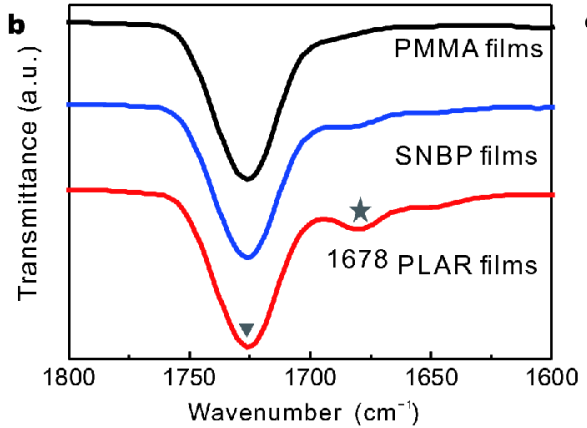
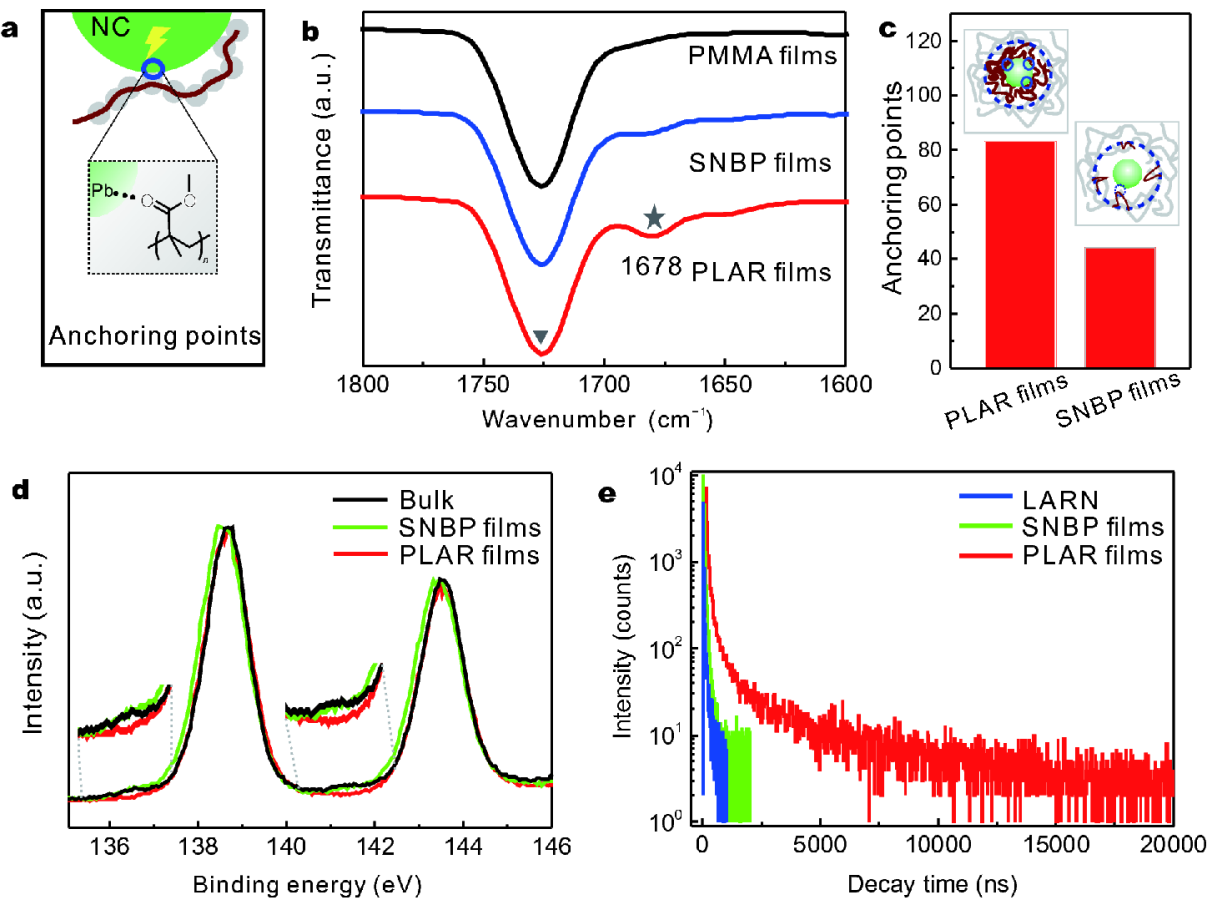

Figure 2 Chemical bond interactions between the $\mathrm{MAPbBr}_{3} \mathrm{NCs}$ discrete phase and the PMMA continuous matrix and full coating of the $\mathrm{Pb}$ ion on the NC surfaces. (a) Schematic sketches showing the anchoring point bond interactions between the $\mathrm{C}=\mathrm{O}$ of $\mathrm{PMMA}$ and $\mathrm{Pb}$ ion on the MAPbBr${ }_{3} \mathrm{NC}$ surface; (b) expanded fingerprint region for the ester carbonyl groups $(\mathrm{C}=\mathrm{O})$ vibrations from FTIR spectra of the pristine PMMA films, SNBP films and PLAR films; (c) the number of anchoring points of SNBP films and PLAR films, and the insets shows the schematic illustration of dense interface phase with full coating of the $\mathrm{Pb}$ ion for PLAR films and loose interface phase with little coating of the Pb ion for SNBP films; (d) XPS spectra of Pb $4 \mathrm{f}$ of bulk perovskite, SNBP films and PLAR films, respectively; (e) TRPL measurements spectroscopy of LAR-synthesized MAPbBr 3 NCs (LARN), SNBP films and PLAR films, respectively.

space of PMMA chains is restricted by crosslinking points of the $\mathrm{MAPbBr}_{3} \mathrm{NCs}$, resulting in a delayed thermal decomposition.

The high-resolution XPS spectra of $\mathrm{Pb} 4 \mathrm{f}$ illustrate the chemical state of the $\mathrm{Pb}^{2+}$ coated by anchoring points, as shown in Fig. 2d. The small peaks at the lower binding energy of $\mathrm{Pb} 4 \mathrm{f}$ main peaks indicate the existence of ionic $\mathrm{Pb}$ on the $\mathrm{MAPbBr}_{3} \mathrm{NC}$ surfaces [36]. The disappearance of low binding energy peaks in the PLAR films implies that all of $\mathrm{Pb}^{2+}$ on the $\mathrm{NC}$ surfaces fully react with the $\mathrm{C}=\mathrm{O}$ in PMMA. The longer PL lifetimes, the better of perovskite films $[23,37]$. The TRPL in Fig. 2e reveals that PLAR films with average PL lifetimes $\left(\tau_{\text {avg }}\right)$ of $1,117 \mathrm{~ns}$ present the much slower PL decay compared to the control sample (SNBP $60 \mathrm{~ns}$ ). Previous reports about $\mathrm{MAPbBr}_{3}$-polymer composite films formed by a swelling microencapsulation strategy showed $\tau_{\text {avg }}$ ranging from 130 (for $\mathrm{MAPbBr}_{3}$-PS) to $502 \mathrm{~ns}$ (for $\mathrm{MAPbBr}_{3}$-ABS) [22]. Good film quality could be attributed to the fully anchoring $\mathrm{Pb}$ cation with PMMA.

The fully coating by denser interface makes the NC surface passivated and induces high exciton binding en- ergy. PL spectum of the PLAR films shows a width at half maximum (FWHM) $24 \mathrm{~nm}$ (Fig. S11), indicating good color purity. The samples show a Stokes shift of $\sim 39 \mathrm{meV}$ between the absorption band edge and the emission line, implying a direct exciton recombination process [38]. A power law dependence $\left(I \propto L^{k}\right)$ was observed with $k$ values near 1.3 (Fig. S12), agreeing well with the excitonic characteristics of the spontaneous emission in semiconductors [39]. Temperature-dependent PL was performed at temperatures ranging from 300 (RT) to $20 \mathrm{~K}$, and a significant temperature quenching behavior of the absolute PL intensity was observed (Fig. 3a). As shown in Fig. 3b, the PL intensity monotonically decreased with increasing temperature, the corresponding exciton binding energy can be calculated as $210 \mathrm{meV}$. This value is much higher than the thermal ionization energy at RT (about $26 \mathrm{meV}$ ), which ensures the survival of excitons at RT. The high exciton binding energy is beneficial to good nanoparticle dispersion and surface passivation by surrounding polymer chains [23]. The PL spectra of the PLAR films at low temperature display additional emission peaks in the high-energy tail located at 2.58, 2.64 and 

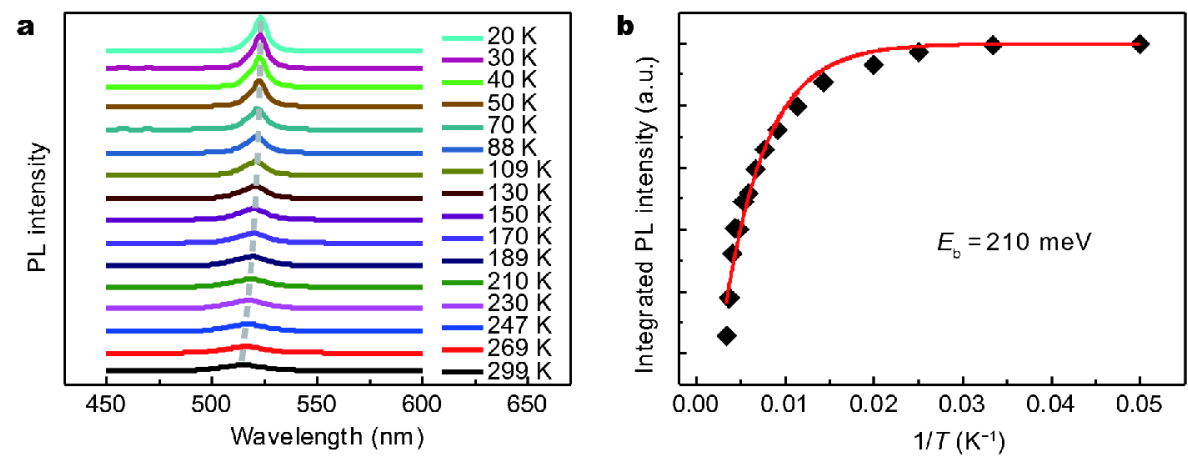

Figure 3 Extionic characteristics of PLAR films with denser interface phase. (a) Temperature-dependent PL spectra taken from 20 to 299 K; (b) PL emission integrated intensity as a function of reciprocal temperature.

$2.71 \mathrm{eV}$ at $20 \mathrm{~K}$ in addition to a dominant emission peak located at $2.37 \mathrm{eV}$, and the high-energy peak disappears with increasing temperature (Fig. S13). The additional high-energy emission peaks are generated by the recombination of charge carriers in MA-ordered orthorhombic domains [40]. The arrangement of MA cations in the ordered domains yields a strong local electric field, resulting in the increased bandgap $E_{\mathrm{g}}$ of the MA-ordered orthorhombic domains (Stark-like effect). A $\Delta E_{\mathrm{g}}$ of $210 \mathrm{meV}$ is observed, which is about 3 times higher than that of bulk $\mathrm{MAPbBr}_{3}$ [40]. This can be ascribed to the polar thermoplastic PMMA resin (dipole moment of 1.3 D) packing the MA, which makes MA exhibit a much larger dipole moment, enhancing the intensity of the Stark-like effect.

The PLAR films show high stability against water and heat, which was evaluated by a long-term direct immersed-water test with periodical PL monitor under UV illumination (Fig. 4a and Supplementary movie SI). After 90 days, the fluorescence intensity still retains $60 \%$, with $15 \%$ decay of PL quantum yield (PLQY) (Fig. S14), despite the full penetration of water into the PMMA film following Fickian diffusion [41]. When the PLAR films were tested in nearly boiling water at $90^{\circ} \mathrm{C}$ (higher than the $T_{\mathrm{g}}$ of the films), PLAR films still exhibit the highest luminescent retention rate (Fig. $4 \mathrm{~b}$ and Fig. S15), compared to SNBP films, SPNC film, and NTSP film, which can be attributed to the denser PMMA phase around NC surface in PLAR films. The continuous PMMA interface layers create a full coating on the $\mathrm{MAPbBr}_{3} \mathrm{NC}$ surface to avoid the direct attack of water, effectively retarding the diffusion of water to the NCs.

The degradation of PLAR-synthesized $\mathrm{MAPbBr}_{3} \mathrm{NC}$ composite films shows two stages, Stage I and Stage II, during the hydrothermal stability test (Fig. 4b). During both stages, the remaining PL brightness under UV illu- mination, composition content, morphology and crystal structure of the films were also characterized. The color of the PLAR films changes from bright green to light green and eventually to dark from Stage I to Stage II with increasing incubation time at $90^{\circ} \mathrm{C}$, and the decreasing of $\mathrm{Br}$ at the surface is much faster than that of $\mathrm{Pb}$ (Fig. 4c, d). The top surface of composite film changed from smooth (Stage I) to coarse porous (Stage II), due to water diffusion, swelling of PMMA into pores [42]. Meanwhile, the crystal structures of the two stages were detected by XRD. The peak at $21.47^{\circ}$ continued to increase, and eventually became dominant, and additional peaks appeared at $23.7^{\circ}, 34.2^{\circ}$ and $39.6^{\circ}$, corresponding to the (111), (031) and (311) planes of $\mathrm{PbBr}_{2}$ (Fig. S16), indicating the formation of $\mathrm{PbBr}_{2}$. With PMMA swelling under the hydrothermal condition, water molecules eventually permeate into the perovskite lattice, triggering the detachment of the $\mathrm{Br}$ anion from the perovskite surface. Hydration of $\mathrm{MAPbBr}_{3}$ is followed by the loss of $\mathrm{CH}_{3} \mathrm{NH}_{3}^{-}$and $\mathrm{Br}^{-}$and then the formed $\mathrm{PbBr}_{2}$ is left in the PMMA matrix due to the interaction between the $\mathrm{C}=\mathrm{O}$ of PMMA and the $\mathrm{Pb}$ ion in the interface phase, which is evidenced by minimum lead leakage in the PLAR films (Fig. 4e).

\section{CONCLUSIONS}

In summary, we demonstrated $\mathrm{MAPbBr}_{3} \mathrm{NC}$-embedded PMMA to form framework composite films by a facile PMMA-introduced LAR approach. Due to the interactions between the carbonyl groups of PMMA and the $\mathrm{Pb}$ ions on the NC surface, the introduction of PMMA into LAR generates long-term stability against water. The resulting dense interface effectively retards the diffusion of water into the perovskite NCs, which promotes the overall stability against water and heat. We believe that this facile PLAR approach will open a new avenue based 

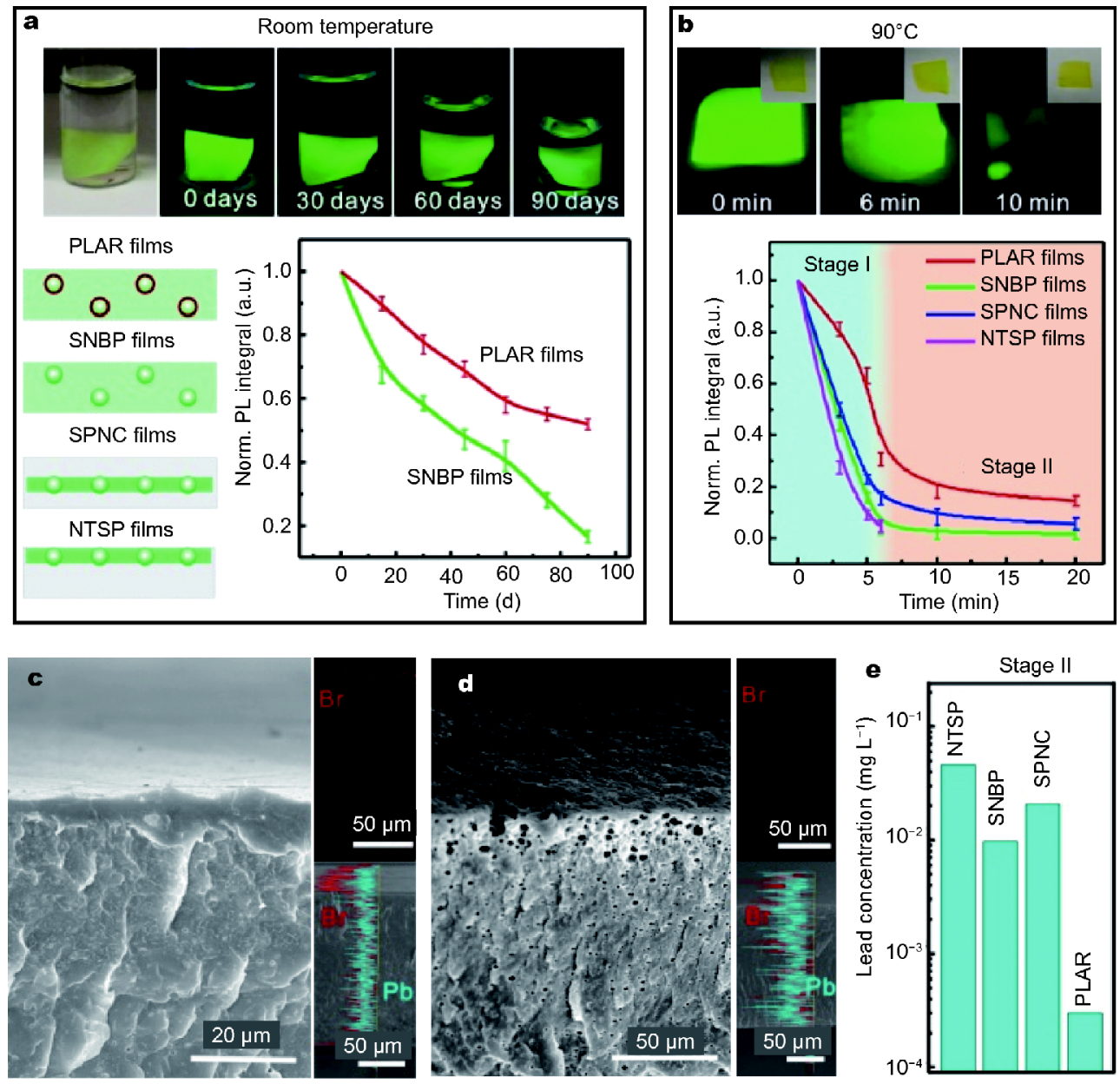

Figure 4 The stability against water and the degradation processes of perovskite. (a) Photographs of PLAR films immersed in water at room temperature were taken under UV irradiation at different time, and the corresponding PL intensity. (b) Photographs of PLAR films treated at $90^{\circ} \mathrm{C}$ (higher than the $T_{\mathrm{g}}$ of PLAR films), and the PL spectra of PLAR films, SNBP films, SPNC films and NTSP films. The color of films changes from bright green at Stage I (c) to dark at Stage II (d), and the cross-section SEM images, element mapping images and EDS line scanning profiles of Br and $\mathrm{Pb}$ are shown in (c) and (d). (e) Lead concentrations of the respective PLAR, SNBP, SPNC and NTSP films at Stage II were analyzed by ICP-OES.

on using polymers as frameworks with functional group to allow coating special atoms, lowering surface defects and enhancing stability.

Received 21 August 2017; accepted 24 October 2017; published online 25 January 2018

1 Kojima A, Teshima K, Shirai Y, et al. Organometal halide perovskites as visible-light sensitizers for photovoltaic cells. J Am Chem Soc, 2009, 131: 6050-6051

2 Lee MM, Teuscher J, Miyasaka T, et al. Efficient hybrid solar cells based on meso-superstructured organometal halide perovskites. Science, 2012, 338: 643-647

3 Yin $\mathrm{X}, \mathrm{Xu} \mathrm{Z}$, Guo Y, et al. Ternary oxides in the $\mathrm{TiO}_{2}-\mathrm{ZnO}$ system as efficient electron-transport layers for perovskite solar cells with efficiency over 15\%. ACS Appl Mater Interfaces, 2016, 8: 2958029587

4 Yin X, Guo Y, Xue Z, et al. Performance enhancement of per- ovskite-sensitized mesoscopic solar cells using Nb-doped $\mathrm{TiO}_{2}$ compact layer. Nano Res, 2015, 8: 1997-2003

5 Cho $\mathrm{H}$, Jeong $\mathrm{SH}$, Park $\mathrm{MH}$, et al. Overcoming the electroluminescence efficiency limitations of perovskite light-emitting diodes. Science, 2015, 350: 1222-1225

6 Tan ZK, Moghaddam RS, Lai ML, et al. Bright light-emitting diodes based on organometal halide perovskite. Nat Nanotech, 2014, 9: 687-692

7 Qin X, Dong H, Hu W. Green light-emitting diode from bromine based organic-inorganic halide perovskite. Sci China Mater, 2015, 58: 186-191

8 Zhu $\mathrm{H}$, Fu Y, Meng F, et al. Lead halide perovskite nanowire lasers with low lasing thresholds and high quality factors. Nat Mater, 2015, 14: 636-642

9 Dou L, Yang YM, You J, et al. Solution-processed hybrid perovskite photodetectors with high detectivity. Nat Commun, 2014, 5: 5404

10 Xue M, Zhou H, Xu Y, et al. High-performance ultraviolet-visible 
tunable perovskite photodetector based on solar cell structure. Sci China Mater, 2017, 60: 407-414

11 Leijtens T, Eperon GE, Noel NK, et al. Stability of metal halide perovskite solar cells. Adv Energy Mater, 2015, 5: 1500963

12 Rong Y, Liu L, Mei A, et al. Beyond efficiency: the challenge of stability in mesoscopic perovskite solar cells. Adv Energy Mater, 2015, 5: 1501066

13 Berhe TA, Su WN, Chen CH, et al. Organometal halide perovskite solar cells: degradation and stability. Energy Environ Sci, 2016, 9: 323-356

14 Wei J, Shi C, Zhao Y, et al. Potentials and challenges towards application of perovskite solar cells. Sci China Mater, 2016, 59: 769-778

15 Mosconi E, Azpiroz JM, De Angelis F. Ab initio molecular dynamics simulations of methylammonium lead iodide perovskite degradation by water. Chem Mater, 2015, 27: 4885-4892

16 Haruyama J, Sodeyama K, Han L, et al. Termination dependence of tetragonal $\mathrm{CH}_{3} \mathrm{NH}_{3} \mathrm{PbI}_{3}$ surfaces for perovskite solar cells. J Phys Chem Lett, 2014, 5: 2903-2909

17 Li B, Fei C, Zheng K, et al. Constructing water-resistant $\mathrm{CH}_{3} \mathrm{NH}_{3}$ $\mathrm{PbI}_{3}$ perovskite films via coordination interaction. J Mater Chem A, 2016, 4: 17018-17024

18 Li X, Ibrahim Dar M, Yi C, et al. Improved performance and stability of perovskite solar cells by crystal crosslinking with alkylphosphonic acid $\omega$-ammonium chlorides. Nat Chem, 2015, 7: 703-711

19 Guarnera S, Abate A, Zhang W, et al. Improving the long-term stability of perovskite solar cells with a porous $\mathrm{Al}_{2} \mathrm{O}_{3}$ buffer layer. J Phys Chem Lett, 2015, 6: 432-437

20 Conings B, Drijkoningen J, Gauquelin N, et al. Intrinsic thermal instability of methylammonium lead trihalide perovskite. Adv Energy Mater, 2015, 5: 1500477

21 Habisreutinger SN, Leijtens T, Eperon GE, et al. Carbon nanotube/ polymer composites as a highly stable hole collection layer in perovskite solar cells. Nano Lett, 2014, 14: 5561-5568

22 Huang S, Li Z, Kong L, et al. Enhancing the stability of $\mathrm{CH}_{3} \mathrm{NH}_{3}$ $\mathrm{PbBr}_{3}$ quantum dots by embedding in silica spheres derived from tetramethyl orthosilicate in "waterless" toluene. J Am Chem Soc, 2016, 138: 5749-5752

23 Di D, Musselman KP, Li G, et al. Size-dependent photon emission from organometal halide perovskite nanocrystals embedded in an organic matrix. J Phys Chem Lett, 2015, 6: 446-450

24 Zhou Q, Bai Z, Lu WG, et al. In situ fabrication of halide perovskite nanocrystal-embedded polymer composite films with enhanced photoluminescence for display backlights. Adv Mater, 2016, 28: 9163-9168

25 Wang Y, He J, Chen H, et al. Ultrastable, highly luminescent organic-inorganic perovskite-polymer composite films. Adv Mater, 2016, 28: 10710-10717

26 Raja SN, Bekenstein Y, Koc MA, et al. Encapsulation of perovskite nanocrystals into macroscale polymer matrices: enhanced stability and polarization. ACS Appl Mater Interfaces, 2016, 8: 3552335533

27 Chen K, Schünemann S, Tüysüz $H$. Preparation of waterproof organometal halide perovskite photonic crystal beads. Angew Chem Int Ed, 2017, 56: 6548-6552

28 Tannenbaum R, Zubris M, David K, et al. FTIR characterization of the reactive interface of cobalt oxide nanoparticles embedded in polymeric matrices. J Phys Chem B, 2006, 110: 2227-2232

29 Huang H, Susha AS, Kershaw SV, et al. Control of emission color of high quantum yield $\mathrm{CH}_{3} \mathrm{NH}_{3} \mathrm{PbBr}_{3}$ perovskite quantum dots by precipitation temperature. Adv Sci, 2015, 2: 1500194

30 Zhang F, Zhong $\mathrm{H}$, Chen C, et al. Brightly luminescent and colortunable colloidal $\mathrm{CH}_{3} \mathrm{NH}_{3} \mathrm{PbX}_{3}(\mathrm{X}=\mathrm{Br}, \mathrm{I}, \mathrm{Cl})$ quantum dots: potential alternatives for display technology. ACS Nano, 2015, 9: 4533-4542

31 Tannenbaum R, King S, Lecy J, et al. Infrared study of the kinetics and mechanism of adsorption of acrylic polymers on alumina surfaces. Langmuir, 2004, 20: 4507-4514

32 Ciprari D, Jacob K, Tannenbaum R. Characterization of polymer nanocomposite interphase and its impact on mechanical properties. Macromolecules, 2006, 39: 6565-6573

33 Zhu F, Men L, Guo Y, et al. Shape evolution and single particle luminescence of organometal halide perovskite nanocrystals. ACS Nano, 2015, 9: 2948-2959

34 Konstadinidis K, Thakkar B, Chakraborty A, et al. Segment level chemistry and chain conformation in the reactive adsorption of poly(methyl methacrylate) on aluminum oxide surfaces. Langmuir, 1992, 8: 1307-1317

35 Zeng R, Rong MZ, Zhang MQ, et al. Interfacial interaction in Ag/ polymer nanocomposite films. J Mater Sci Lett, 2001, 20: 1473 1476

$36 \mathrm{Li} \mathrm{X}, \mathrm{Wu} \mathrm{Y}$, Zhang S, et al. $\mathrm{CsPbX}_{3}$ quantum dots for lighting and displays: room-temperature synthesis, photoluminescence superiorities, underlying origins and white light-emitting diodes. Adv Funct Mater, 2016, 26: 2435-2445

37 de Quilettes DW, Vorpahl SM, Stranks SD, et al. Impact of microstructure on local carrier lifetime in perovskite solar cells. Science, 2015, 348: 683-686

38 Zheng $\mathrm{K}$, Zhu Q, Abdellah M, et al. Exciton binding energy and the nature of emissive states in organometal halide perovskites. J Phys Chem Lett, 2015, 6: 2969-2975

39 Schmidt T, Lischka K, Zulehner W. Excitation-power dependence of the near-band-edge photoluminescence of semiconductors. Phys Rev B, 1992, 45: 8989-8994

40 Dar MI, Jacopin G, Meloni S, et al. Origin of unusual bandgap shift and dual emission in organic-inorganic lead halide perovskites. Sci Adv, 2016, 2: e1601156

41 Shobhana E. X-ray diffraction and UV-visible studies of PMMA thin films. Int J Modern Eng Res, 2012, 2: 1092-1095

42 N'Diaye M, Pascaretti-Grizon F, Massin P, et al. Water absorption of poly(methyl methacrylate) measured by vertical interference microscopy. Langmuir, 2012, 28: 11609-11614

Acknowledgements This work was supported by the Thousand Young Talents Program, and the National Natural Science Foundation of China (21422507, 21635002 and 21321003).

Author contributions Wang T and Li X designed the research. Li X, Xue $\mathrm{Z}$ and Huang $\mathrm{C}$ performed the experiments and analyzed results. Luo D and Liu L performed optical spectroscopy measurements. Qiao X and Liu C carried samples stability test. Li X wrote the manuscript. All authors participated in the general discussion of the paper.

Conflict of interest The authors declare that they have no conflict of interest.

Supplementary information Experimental details and supporting data are available in the online version of the paper. 


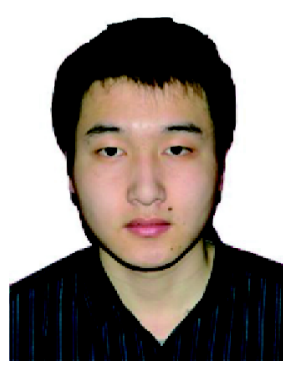

Xiao Li is now a Master candidate in materials science and engineering at the North University of China. His current research interests are focused on the synthesis of perovskite nanomaterials and polymer composites.

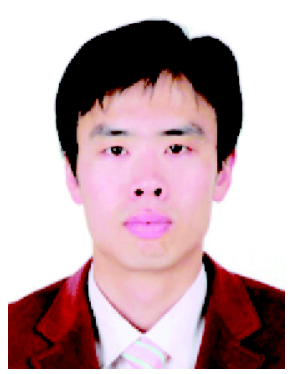

Tie Wang is a professor at the Institute of Chemistry, Chinese Academy of Sciences (ICCAS). He received his PhD (2007) from Changchun Institute of Applied Chemistry. He joined the ICCAS with the award of the "Thousand Youth Talents Plan" in 2013. His research is focused on the assemblies and applications of nanoparticles.

\section{一种高分子保护的铅卤钻钛矿纳米晶}

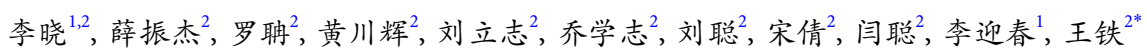

摘要 有机-无机杂化铅卤钙钛矿易于加工、带隙可调、电荷转移速率高, 是一种具有广泛应用前景的新型光电半导体材料. 在潮湿空气 中的稳定性是钲钛矿实现产业化应用函待解决的问题. 本文介绍了聚甲基丙烯酸甲酯作为配体利用配体辅助再沉淀实现了钙钛矿纳米晶 的聚合物包裹. 聚合物作为疏水性骨架通过功能性酯羰基与钙铁矿表面铅化学键合实现了表面铅位点的全覆盖, 有效阻止该位点被水分 子占据, 形成的紧密界面层有效延缓水分子扩散到钻钛矿纳米晶中. 制备的薄膜表现出超高的浸水稳定性. 